\title{
Anticoncepción hormonal, aspectos sociodemográficos y de la conducta como factores de riesgo para cáncer de cuello uterino en un hospital en Ica, Perú.
}

\author{
Hormonal contraception, sociodemographic and behavioral \\ aspects as risk factors for cervical cancer in a hospital in Ica, Perú.
}

Kuroki-Yshii Ana, ${ }^{1, a}$, Franco-Kuroki Alejandra, b, Alaba-Tucto Karina ${ }^{3, b}$.

1. Facultad de Medicina, Universidad Nacional "San Luis Gonzaga" de Ica.

2. Dirección Regional de Salud de Ica

3. Dirección Regional de Salud de Ayacucho

a. Medico Gineco-obstetra.

b. Médico cirujano

\section{Correspondencia:}

Ana María Kuroki.

Número de celular: 985566837

Correo Electrónico:

anakuroki15@hotmail.com

Domicilio: Calle girasoles G-12 Residencial la angostura, Ica, Perú.

\section{Contribuciones De Autoría:}

KYA, FKA y ATK : Contribuciones en la concepción y diseño del manuscrito, recolección, análisis e interpretación de los datos, redacción y revisión crítica del contenido del manuscrito y aprobación final del artículo.

Conflicto De Intereses: No declarados.

Financiamiento: Autofinanciado.

\section{Como Citar}

Kuroki-Yshii Ana, Franco-Kuroki Alejandra, Alaba-Tucto Karina. Anticoncepción hormonal, aspectos sociodemográficos y de la conducta como factores de riesgo para cáncer de cuello uterino en un Hospital de Ica. Rev méd panacea 2018; (2): 41-49

Recibido: 17 - 04 - 2018

Aceptado: 17 - 05 - 2018

Publicado: 15 - 08 - 2018

\section{RESUMEN}

Objetivo: Demostrar que la anticoncepción hormonal, los factores sociodemográficos y de la conducta constituyen factores de riesgo para cáncer de cuello uterino en las pacientes atendidas en consultorio externo de Ginecología y Prevención del Cáncer en el Hospital Santa María del Socorro de Ica entre enero 2015 y diciembre 2017. Materiales y métodos: Estudio observacional, retrospectivo, transversal, descriptivo, analítico. Resultados: La prevalencia de cáncer de cuello uterino fue de 13.7\% (13 pacientes de cada 100 atendidas tuvieron cáncer de cuello uterino). Los resultados muestran que hubo mayor prevalencia en las mujeres mayores de 35 años, casadas o convivientes, sin estudios o con primaria completa, multíparas, con inicio de vida sexual después de los 14 años y no usuarias de anticonceptivos hormonales. No se encontró significancia estadística en ninguno de los factores mencionados. Conclusiones: No se demostró asociación entre anticoncepción hormonal y cáncer de cuello uterino. Los factores sociodemográficos y de la conducta algunos con mayor prevalencia no fueron estadísticamente significativos.

Palabras clave: Neoplasias del cuello uterino, Anticoncepción, Factores de riesgo. (Fuente: DeCS BIREME)

\begin{abstract}
Objective: To demonstrate that hormonal contraception, sociodemographic and behavioral factors constitute risk factors for cervical cancer in patients treated at the gynecology and cancer prevention clinic at the Santa Maria del Socorro Hospital in Ica between January 2015 and December 2017. Materials and methods: Observational, retrospective, cross-sectional, analytical descriptive study in a sample of 190 patients chosen at random for convenience in the outpatient gynecology and cancer prevention strategy of the Santa Maria del Socorro Hospital, who underwent an examination. of PAP and presumptive cervical cancer colposcopy and biopsy. Results: The prevalence of cervical cancer was $13.7 \%$ (13 patients out of 100 attended had cervical cancer). The results show that there was a higher prevalence in women older than 35 years, married or cohabiting, without studies or with complete primary, multiparous, with sexual life beginning after 14 years and non-users of hormonal contraceptives. No statistical significance was found in any of the mentioned cases. Conclusions: There was no statistically significant association between hormonal contraception and cervical cancer. Sociodemographic and behavioral factors, although with a higher proportion, were not statistically significant.
\end{abstract}

Keywords: Uterine cervical neoplasms, contraception, Risk factors. (Source: MeSH NLM). 


\section{INTRODUCCIÓN}

El cáncer de cuello uterino (CCU) es un serio problema de salud pública a nivel mundial. Según la OMS, en el mundo se presentan alrededor de 500.000 nuevos casos por año y se producen alrededor de 250.000 muertes por esta causa (1). En América Latina y el Caribe, es la tercera neoplasia maligna más frecuente considerando estadísticamente ambos sexos, y la segunda en las mujeres, con una tasa estandarizada por edad (TEE) de 24 casos por 100,000 mujeres; ubicándose también en segundo lugar en importancia en mortalidad (2).

Según la Liga Contra el Cáncer, los últimos estudios a nivel nacional reportan al cáncer de cuello uterino como la primera causa de muerte en el Perú; con una incidencia de 16 mil casos y ubicándose como la primera causa de morbilidad por cáncer en la mujer peruana y la segunda en Lima3. Cada día entre 12 y 14 mujeres son diagnosticadas con cáncer de cuello uterino y 6 o 7 mueren por la falta de una detección oportuna (3).

Es por ello que durante la última década aumentó la investigación mundial en torno a la mejor comprensión de la historia natural de la enfermedad, su relación con la infección por Virus Papiloma Humano (VPH) y los factores condicionantes de la misma. Los estudios al respecto incluyen la determinación de la prevalencia de la infección según genotipos y estudios de seguimiento de la asociación con diversos factores, como: métodos anticonceptivos, comportamiento sexual, dieta, tabaquismo, genéticos e inmunosupresión (4). Sin embargo, la naturaleza exacta de esta relación no está clara todavía.

En nuestra Región no existen una casuística sincerada ni actualizada al respecto $(5,6)$ y la información de probable causalidad, se establece en relación a los antecedentes de otras latitudes.

El aumento considerable de casos de cáncer de cuello uterino a nivel mundial, nacional y regional fue el motivo por el cual se eligió este trabajo de investigación y siendo el método hormonal una de las formas más utilizadas de anticoncepción consideramos importante conocer la relación existente entre anticoncepción hormonal un método de planificación familiar de gran uso en nuestra población y el riesgo de padecer cáncer de cuello uterino. De igual forma, se buscó identificar qué factores sociodemográficos y de la conducta representan factores de riesgo para la enfermedad. A la luz de los resultados obtenidos se intenta brindar un aporte a la prevención mediante el conocimiento de la relación que existe entre anticoncepción hormonal, factores socio demográficos y de la conducta (edad de inicio de las relaciones sexuales, promiscuidad, edad, pobreza) y el cáncer de cuello uterino, de tal manera que podamos contribuir a que los organismos responsables del sector salud diseñen escenarios que mejoren la estrategia de prevención y control de esta enfermedad.

\section{MATERIALES Y MÉTODOS}

Se realizó una investigación de tipo observacional, descriptiva, retrospectiva, analítica y de corte transversal.

La población accesible, estuvo constituida por las mujeres que acudieron al consultorio de Ginecología o al Centro de Despistaje y Prevención de Cáncer del Hospital Santa María del Socorro de Ica, con sospecha de cáncer de cuello uterino y a quienes se les practicó examen de Papanicolaou, colposcopía y biopsia para confirmación del diagnóstico, desde enero del 2015 hasta diciembre del 2017.

La muestra conformada inicialmente por 212 pacientes, cuyos datos se encontraban en la unidad de análisis representada por sus propias historias clínicas o sus fichas de recolección del Programa de Prevención de Cáncer, las que sometidas a los criterios de inclusión y exclusión y, debido a falta de datos o incongruencia en los diagnósticos, se realizó la exclusión de 22 de ellas, quedando constituida la muestra finalmente por 190 pacientes, que es la que se depositó en nuestra base de datos.

\section{CRITERIOS DE INCLUSION:}

- Todas las historias clínicas de pacientes atendidas en la consulta externa de Ginecología o al Centro de Despistaje y Prevención de Cáncer del Hospital Santa María del Socorro de Ica, con sospecha de cáncer de cuello uterino, que cuenten con los datos sobre aspectos sociodemográficos, de la conducta y uso de anticoncepción hormonal al ser sometidas a los criterios de calidad.

- Todas las historias clínicas de pacientes atendidas en la consulta externa de Ginecología o al Centro de Despistaje y Prevención de Cáncer del Hospital Santa María del Socorro de Ica, con sospecha de cáncer de cuello uterino y a quienes se les practicó examen de Papanicolaou, colposcopía o biopsia para confirmación del diagnóstico, debidamente confeccionadas, contando con los resultados de los exámenes solicitados.

\section{CRITERIOS DE EXCLUSION:}

- Pacientes cuyas historias clínicas o fichas de recolección de datos estén incompletas o incongruentes con la clasificación de la patología.

- Pacientes con diagnóstico de cáncer de otra localización y/o no pertenezcan al área de estudio.

Los datos obtenidos de las unidades de análisis, fueron llevados a una ficha Ad-hoc, confeccionada por los investigadores, cuya base de datos se depositó en una hoja de cálculo en el programa Microsoft Excel 2010, dicha información conservó el anonimato de los pacientes, utilizando un código de identificación conocido sólo por los investigadores. 
Se verificó que a los pacientes se les hubiera practicado un examen de Papanicolaou, con uniformización de los criterios de diagnóstico citológico a la clasificación de Bethesda: Lesión intraepitelial de bajo grado (SIL 1) y Lesión intraepitelial de alto grado (SIL 2); a los pacientes con lesión sospechosa se sometieron a colposcopia y biopsia, se verificó el informe del resultado para cáncer de cuello uterino.

La información recolectada incluyó variables referidas a anticoncepción hormonal, características sociodemográficas y de la conducta, donde se consignaron los datos obtenidos teniendo en cuenta los criterios de inclusión y exclusión, establecidos con antelación y consignados en la ficha de recolección.

Haciendo uso del programa SPSS 23.0 para MS Windows, se procedió a la elaboración de la base y transferencia de la información recolectada, previo control de calidad. Con la ayuda del programa se hizo el análisis descriptivo de todas las variables obteniendo frecuencias simples y proporciones. El análisis bivariado se realizó mediante el test del Chi cuadrado para las variables categóricas y la prueba de t de student para las variables numéricas según correspondía. Se asumió una relación significativa si $p<0.05$.

Asimismo, se empleó el Odds ratio (OR) -calculado mediante la razón de productos cruzados con los datos de la tabla de contingencia de $2 \times 2$ para determinar la relación de riesgo.

En la prueba Chi cuadrado se rechazará la hipótesis nula si el valor esperado de chi cuadrado es igual o mayor que el valor crítico, mientras que el OR será calculado mediante el cálculo de la razón de productos cruzados con los datos de la tabla de contingencia.

\section{RESULTADOS}

Tabla 1: La edad y su asociación con la aparición de cáncer de cuello uterino.

\begin{tabular}{ccccccc} 
& \multicolumn{4}{c}{ CCU } \\
& & & Presenta & No presenta & Total \\
\hline \multirow{2}{*}{ Edad } & Mayor de 35 años & $f$ & 26 & 144 & 170 \\
& & $\%$ & $15,30 \%$ & $84,70 \%$ & $100,00 \%$ \\
& Menor o igual a 35 años & $f$ & 0 & 20 & 20 & $100,00 \%$ \\
\hline \multirow{2}{*}{ Total } & & $\%$ & $0,00 \%$ & $100,00 \%$ & 164 & 190 \\
& & $f$ & 26 & $86,30 \%$ & $100,00 \%$ \\
\hline
\end{tabular}

Fuente: Datos de la hoja de recolección de información ( $p$ valor: 0,060)

La prevalencia de cáncer de cuello uterino es mayor en las mujeres cuya edad sobrepasa los 35 años (15.3\%) en comparación con las mujeres cuyas edades son iguales o menores a 35 años (0.0\%), rubro en el cual no se halló ningún caso. Sin embargo, la prueba chi cuadrado indica que no hay diferencia estadísticamente significativa entre ambas proporciones.

Tabla 2: Análisis descriptivo del tiempo de inmovilidad en la prueba de nado forzado

\begin{tabular}{|c|c|c|c|c|c|}
\hline & & \multicolumn{4}{|c|}{$\mathbf{C C U}$} \\
\hline & & & Presenta & No presenta & Total \\
\hline \multirow{4}{*}{ Estado Civil } & \multirow{2}{*}{ Soltera - Viuda } & $f$ & 6 & 49 & 55 \\
\hline & & $\%$ & $10,90 \%$ & $89,10 \%$ & $100,00 \%$ \\
\hline & \multirow{2}{*}{ Casada - Conviviente } & $f$ & 20 & 115 & 135 \\
\hline & & $\%$ & $14,80 \%$ & $85,20 \%$ & $100,00 \%$ \\
\hline \multirow{2}{*}{ Total } & & $f$ & 26 & 164 & 190 \\
\hline & & $\%$ & $13,70 \%$ & $86,30 \%$ & $100,00 \%$ \\
\hline
\end{tabular}

Fuente: Datos de la hoja de recolección de información (p-valor: 0,477)

Las mujeres casadas o convivientes presentan una prevalencia de 14.8\% de cáncer de cuello uterino, cifra mayor al 10.9\% hallado en las mujeres solteras o viudas. No se halló significancia estadística para la diferencia de proporciones al aplicar la prueba del chi cuadrado. 
Tabla 3: El grado de instrucción y su asociación con la aparición de cáncer de cuello uterino

\begin{tabular}{cccccc} 
& & \multicolumn{3}{c}{ CCU } \\
& & & Presenta & No presenta & Total \\
\hline \multirow{2}{*}{$\begin{array}{c}\text { Grado de } \\
\text { instrucción }\end{array}$} & Sin estudios o Primaria & $f$ & 11 & 40 & 51 \\
& Secundaria completa o & $\mathrm{f}$ & 15 & 124 & 139 \\
\hline \multirow{2}{*}{ Total } & Superior & $\%$ & $10,80 \%$ & $89,20 \%$ & $100,00 \%$ \\
\hline
\end{tabular}

Fuente: Datos de la hoja de recolección de información (p-valor: 0,055)

Se observa que las mujeres sin estudios o con primaria completa (21.6\%) presentan mayor prevalencia de cáncer de cuello uterino que las mujeres con secundaria completa o superior (10.8\%). La prueba chi cuadrado indica que no hay diferencia estadísticamente entre estas proporciones.

Tabla 4: El número de parejas y su asociación con la presencia de cáncer de cuello uterino.

\begin{tabular}{|c|c|c|c|c|c|}
\hline & & \multicolumn{4}{|c|}{ CCU } \\
\hline & & & Presenta & No presenta & Total \\
\hline \multirow{4}{*}{$\begin{array}{c}\text { No }^{\circ} \\
\text { Parejas }\end{array}$} & \multirow{2}{*}{ Dos o más parejas } & $f$ & 16 & 101 & 117 \\
\hline & & $\%$ & $13,70 \%$ & $86,30 \%$ & $100,00 \%$ \\
\hline & \multirow{2}{*}{ Una pareja } & $f$ & 10 & 63 & 73 \\
\hline & & $\%$ & $13,70 \%$ & $86,30 \%$ & $100,00 \%$ \\
\hline \multirow{2}{*}{ Total } & & $f$ & 26 & 164 & 190 \\
\hline & & $\%$ & $13,70 \%$ & $86,30 \%$ & $100,00 \%$ \\
\hline
\end{tabular}

Fuente: Datos de la hoja de recolección de información (p-valor: 0,996)

Los resultados del presente estudio muestran que la prevalencia de cáncer de cuello uterino en la muestra analizada fue similar tanto en las mujeres con dos o más parejas como en aquellas que indicaron haber tenido una pareja (13.7\%). La prueba chi cuadrado indica que no hay diferencia estadísticamente significativa entre ambas proporciones.

Tabla 5: La edad de inicio de las relaciones sexuales y su asociación con cáncer de cuello uterino.

\begin{tabular}{|c|c|c|c|c|c|}
\hline & & & \multicolumn{2}{|c|}{$\mathbf{C C U}$} & \multirow[b]{2}{*}{ Total } \\
\hline & & & Presenta & No presenta & \\
\hline \multirow{4}{*}{$\begin{array}{c}\text { Edad de inicio } \\
\text { de las RS }\end{array}$} & \multirow{2}{*}{14 años o menos } & $f$ & 2 & 16 & 18 \\
\hline & & $\%$ & $11,10 \%$ & $88,90 \%$ & $100,00 \%$ \\
\hline & \multirow{2}{*}{ Más de 14 años } & $f$ & 24 & 148 & 172 \\
\hline & & $\%$ & $14,00 \%$ & $86,00 \%$ & $100,00 \%$ \\
\hline \multirow{2}{*}{ Total } & & $f$ & 26 & 164 & 190 \\
\hline & & $\%$ & $13,70 \%$ & $86,30 \%$ & $100,00 \%$ \\
\hline
\end{tabular}

Fuente: Datos de la hoja de recolección de información ( $p$-valor: 0,738)

Las mujeres que refieren haber iniciado su vida sexual a los 14 años o menos (11.1\%) presentan menor prevalencia de cáncer de cuello uterino que aquellas que lo hicieron más alla de los 14 años (14\%) respectivamente; sin embargo, la prueba chi cuadrado indica que no hay significancia estadística entre estas proporciones. 
Tabla 6: La multiparidad y su asociación con la presencia de cáncer de cuello uterino.

\begin{tabular}{cccccc} 
& & \multicolumn{3}{c}{ CCU } \\
& & & Presenta & No presenta & Total \\
\hline \multirow{3}{*}{ Multiparidad } & Multípara & $f$ & 21 & 114 & 135 \\
& & $\%$ & $15,60 \%$ & $84,40 \%$ & $100,00 \%$ \\
& \multirow{2}{*}{ No multípara } & $f$ & 5 & 50 & 55 \\
& & $\%$ & $9,10 \%$ & $90,90 \%$ & $100,00 \%$ \\
\hline \multirow{2}{*}{ Total } & $\mathrm{f}$ & 26 & 164 & 190 \\
& & $\%$ & $13,70 \%$ & $86,30 \%$ & $100,00 \%$ \\
\hline
\end{tabular}

Fuente: Datos de la hoja de recolección de información ( $p$-valor: 0,240)

Se observa que las mujeres multíparas (15.6\%) presentan mayor prevalencia de cáncer de cuello uterino que las mujeres que no presentan multiparidad (9.1\%); sin embargo, la prueba chi cuadrado indica que no hay diferencia estadísticamente significativa entre ambas proporciones.

Tabla 7: El uso de anticonceptivos hormonales y su asociación con la presencia de cáncer de cuello uterino.

\begin{tabular}{cccccc} 
& & \multicolumn{4}{c}{ CCU } \\
& & & Presenta & No presenta & Total \\
Uso de & \multirow{2}{*}{$\begin{array}{c}\text { Usa } \\
\text { anticoncepción }\end{array}$} & $\%$ & 13 & 102 & 115 \\
hormonal & \multirow{2}{*}{ No usa } & $\mathrm{f}$ & $13,30 \%$ & $88,70 \%$ & $100,00 \%$ \\
& & $\%$ & $17,30 \%$ & $82,70 \%$ & $100,00 \%$ \\
\hline \multirow{2}{*}{ Total } & $\mathrm{f}$ & 26 & 164 & 190 \\
& & $\%$ & $13,70 \%$ & $86,30 \%$ & $100,00 \%$ \\
\hline
\end{tabular}

Fuente: Datos de la hoja de recolección de información (p-valor: 0,237)

En la muestra del estudio las mujeres que no usan anticonceptivos hormonales presentan mayor prevalencia de cáncer de cuello uterino (17.7\%) que las mujeres que usan estos anticonceptivos hormonales (11.3\%); sin embargo, la prueba chi cuadrado indica que no hay diferencia estadísticamente significativa entre estas dos proporciones.

\section{DISCUSIÓN}

Nuestra investigación trató de uniformizar los diferentes resultados a la clasificación de Bethesda, actualmente recomendada en los programas de Ginecología y Prevención de Cáncer (7,8). Luego se hizo la separación de los casos estrictos de cáncer in situ de las lesiones pre-malignas restantes para unirlos a los casos de cáncer invasor y confrontar los resultados con el resto de lesiones pre-malignas y aquellos casos sospechosos que resultaron normales tras la toma de Papanicolaou para establecer la diferencia de proporciones entre pacientes con cáncer y pacientes que no tenían cáncer, asociados a cada factor de riesgo.

La etiología del cáncer de cuello uterino se relaciona con múltiples causas. El VPH es una causa necesaria, pero no suficiente, para desarrollar cáncer de cuello uterino (1, $2,7,9,10)$. De acuerdo a lo reportado por diversos estudios se estima que los factores de riesgo asociados a esta enfermedad pueden ser: edad, consumo de alcohol y tabaco, uso de anticonceptivos, inicio temprano de vida sexual, número de parejas sexuales, factores genéticos, edad del primer embarazo, hábitos sexuales, condiciones socioeconómicas, analfabetismo, deficiencias nutricionales, ausencia de antecedentes en la citología vaginal $(1,2,7,9,10)$. Nuestro trabajo de tesis recogió algunas de las variables mencionadas y analiza la posible asociación entre el I uso de anticonceptivos hormonales, factores sociodemográficos y de la conducta como factores de riesgo para el cáncer de cuello uterino.

Referente a los factores sociodemográficos asociados a la presencia de cáncer de cuello uterino, nuestra investigación halló que la prevalencia de esta enfermedad fue mayor en aquellas mujeres que tenían más de 35 años de edad, sin significancia estadística ( $p$ : 0,060 ). Esta tendencia coincide con las observaciones 
hechas por otros investigadores $(5,6,11,12,13,14,15)$, quienes refieren que a mayor edad existe un mayor riesgo de padecer la enfermedad. Es el caso de Vega, D. en México (2004), quien en su estudio titulado "Factores de riesgo para cáncer cervicouterino en mujeres de Matamoros, Tamaulipas" uno de los factores con mayor prevalencia fue la edad mayor de 35 años (53.4\%)(9). En nuestro estudio, 15 de cada 100 pacientes con cáncer de cuello uterino tenían una edad mayor a los 35 años.

En cuanto al estado civil, debemos señalar que algunas investigaciones como la de García Minaya (16) entre otras (12), revelan que las mujeres solteras, viudas o divorciadas tienen más riesgo de infectarse con el VPH dado que tienen un mayor número de compañeros sexuales, ya sean permanentes u ocasionales $(16,17)$. Se conoce la fuerte asociación que tiene la presencia de esta infección con el cáncer de cérvix, lo cual contrasta con nuestra investigación donde la presencia de cáncer de cuello uterino se dio en mayor proporción en las mujeres casadas o convivientes. Nuestros resultados coinciden con algunos otros estudios $(5,6,11,13)$, esto probablemente revela la promiscuidad existente de parte de uno o ambos componentes de la pareja, lo que se convierte en una alternativa para las oportunidades de contagio para el VPH. No se halló significancia estadística (p: 0,477).

Existen datos controvertidos acerca de la asociación que se da entre cáncer de cuello uterino y condición social, económica o educativa. Se acepta sin embargo que las mujeres de más bajo nivel social, cultural y educativo, así como los inmigrantes extranjeros y con menores conocimientos generales en salud, tienen mayor incidencia de cáncer de cuello uterino $(13,17,18,19)$. Hay autores que incluso manifiestan que la asociación entre nivel educativo y la neoplasia de cérvix se presenta con mayor frecuencia en pacientes analfabetas o que tienen sólo primaria (18). Nuestro estudio coincide con estas investigaciones y nuestro hallazgo fue que en las pacientes sin estudios o con primaria completa, la prevalencia de cáncer de cuello uterino es mayor que en aquellas que tienen estudios de secundaria completa o superior. Ya refería esto Thulaseedharan y asociados (20), quienes observaron que las mujeres sin estudios resultaron tener un riesgo significativamente mayor de cáncer de cuello uterino. En nuestro trabajo, la diferencia de proporciones no resultó estadísticamente significativa según la prueba del Chi cuadrado $(0,055)$.

La presencia de múltiples compañeros sexuales se relaciona en diversos estudios con la aparición de cáncer de cuello uterino $(5,5,9,13,16,19,21,23,24)$. Según Sotomayor Y. (24) en su investigación: "Conocimiento y actitudes respecto al cáncer cervicouterino en las mujeres atendidas en el Hospital Sabogal", refiere que el 83.9\% de mujeres reportó dos compañeros sexuales. Nuestros hallazgos encuentran similar prevalencia en pacientes que aceptaron haber tenido dos o más parejas, así como en las que refirieron haber tenido una sola pareja (13.7\%). Esto orientaría a una modificación del comportamiento epidemiológico de la enfermedad que habría que demostrar.

Otro factor que resulta importante por su relación con la aparición de cáncer de cuello uterino es la edad de inicio de las relaciones sexuales. Al respecto, Raychaudhuri y Mandal (25) hallaron que en la prevalencia de factores de riesgo fue importante la edad temprana de la primera relación sexual en un 65,6\%. Ortiz R. y asociados (9) refieren que el riesgo de lesión intraepitelial cuando el primer coito se tiene a los 17 años o menos es 2,4 veces mayor que cuando este se tiene a los 21 años. Al respecto, García Minaya16 explica que el inicio temprano de las relaciones implica la aparición de múltiples compañeros sexuales, con el consiguiente riesgo dado por éstos. Se ha demostrado también que en la adolescencia los tejidos cervicouterinos son más susceptibles a la acción de los carcinógenos, y, de hecho, si existe un agente infeccioso relacionado, el tiempo de exposición a este será mucho mayor $(14,26)$. Nuestros resultados no coinciden con estas investigaciones que informan la asociación entre aparición de cáncer con un inicio a edad temprana de las relaciones sexuales $(5,12$, $14,21,22,27,29)$. Nuestro hallazgo revela que la prevalencia de cáncer de cuello uterino en pacientes que refieren haber iniciado su actividad sexual a los 14 o menos años fue menor que en las pacientes que refieren haber iniciado su actividad sexual más allá de los 14 años; las diferencias pueden deberse al punto de corte escogido en las diversas investigaciones.

Respecto a la paridad, la presencia de cáncer de cuello uterino en pacientes multíparas fue mayor que en las mujeres no multíparas, a pesar de no ser estadísticamente significativo, lo cual coincide con los resultados de otros investigadores, quienes señalan a la multiparidad como un factor de riesgo asociado a la presencia de lesiones LPM y CCU $(11,13,14,18,22,26,27,28,29,30-32)$.

La utilización de la anticoncepción hormonal en sus diversas formas, ha sido motivo de opiniones divididas y controversiales, toda vez que algunos afirman que existe relación entre la administración de anticoncepción hormonal asociado a no a la presencia del virus del papiloma humano (VPH) y la aparición de lesión pre maligna y cáncer de cuello uterino. Diversas investigaciones realizadas desde hace más de 15 años sostienen que las hormonas esteroides pueden funcionar como facilitadoras de la replicación y persistencia del virus del papiloma humano, factor determinante para el desarrollo de cáncer de cuello uterino33. Sin embargo, considerar el uso de anticonceptivos hormonales como factor de riesgo para cáncer de cuello uterino es controversial. Los diversos estudios realizados a nivel mundial presentan resultados que difieren entre sí $\quad(11,19,21,26,27,28,33,34)$. 
Nuestros hallazgos a pesar no tener significancia estadística señalan que la proporción de aparición de cáncer de cuello uterino fue mayor en mujeres que no usaban anticoncepción hormonal que en las que sí lo hacían, lo cual aleja la posibilidad para este estudio de poder relacionar la utilización de anticoncepción hormonal y cáncer de cuello uterino tal como lo refieren algunas investigaciones. De otro lado nuestros resultados coinciden con los expuestos en el estudio de Mollinedo y colaboradores (35), donde no se encuentra relación directa entre el empleo de anticonceptivos hormonales y cáncer de cuello uterino. (22.6\%). Shapiro y colaboradores 14 reportan un riesgo nulo al asociar anticonceptivos inyectables (acetato de medroxiprogesterona) con cáncer cervicouterino

\section{REFERENCIAS BIBLIOGRÁFICAS}

1. Nueva guía de la oms para la prevención y el control del cáncer cervicouterino [En línea]. Centro de Prensa de la Organización Mundial de la Salud. Melbourne (Australia), 2014. [Consultado el 05 de marzo de 2018] URL disponible en:

http://www.who.int/mediacentre/news/releases/2014 /preventing-cervical-cancer/es/.

2. Guía de práctica clínica, cáncer de cuello uterino [En línea]. INEN. Lima (Perú), 2011. [Consultado 29 de noviembre de 2016] URL disponible en:

http://www.inen.sld.pe/portal/documentos/pdf/norm as_tecnicas/2011/25042011_cancer_cuello_uterino_19 _04_11.pdf.

3. Cáncer de cuello uterino [En línea]. Liga Contra el Cáncer. Lima (Perú), 2016. [Consultado el 05 de marzo de 2018] URL disponible en:

http://www.ligacancer.org.pe/tiposdecancer.html

4. Castro Romero J.I., Hernández Girón C., Madrid Marín V. La anticoncepción hormonal como factor de riesgo para cáncer cervicouterino: evidencias biológicas, inmunológicas y epidemiológicas [En línea]. México, 2011. [Consultado 29 de noviembre de 2016] URL disponible en:

http://docplayer.es/4128604-La-anticoncepcion-hor monal-como-factor-de-riesgo-para-cancer-cervicout erino-evidencias-biologicas-inmunologicas-y-epidem iologicas.html.

5. Aguilar Melgar J.A., Chacaltana Espino L.M., Mujica Gré R.M. Perfil epidemiológico del cáncer de cérvix. hospital Augusto Hernández Mendoza-Ica 2010-2012 [Tesis de grado]. Ica (Perú): Facultad de Medicina Humana de la Universidad Nacional "San Luis Gonzaga" de Ica. 2013.

6. Pacori Rodrigo F., Anicama Matta J.G., Vilca Yange A. Factores clínico epidemiológicos asociados a cáncer de cuello uterino en el hospital Santa María del Socorro de Ica, 2001-2010 [Tesis de grado]. Ica (Perú): Facultad de Medicina Humana de la Universidad Nacional "San Luis Gonzaga" de Ica. 2012.

7. Guía técnica: Guía de práctica clínica para la prevención y manejo del cáncer de cuello uterino [En invasivo (RR 1.0; IC 95\%: 0.8-1.3). Raychaudhuri y Mandal (25) hallaron que la prevalencia de uso de preservativos y de píldoras como factor de riesgo fue de sólo un 5,4\%. Se plantea que la posible asociación anticonceptivos hormonales y cáncer de cuello uterino hallada en otros estudios tiene probablemente más una relación con el comportamiento sexual y la infección por el virus del papiloma humano (VPH) que por efecto directo; Sasiene hace hincapié en que las mujeres que utilizan la píldora para evitar un embarazo son menos propensas a emplear otros métodos, como el preservativo, que impide en gran medida el contagio de $\mathrm{VPH}$; factor determinante para la aparición de cáncer de cuello uterino $(14,26)$.

línea]. Despacho Viceministerial de Salud Pública. Dirección General de Intervenciones Estratégicas en Salud Pública. Dirección de Prevención y control del Cáncer. MINSA. Lima (Perú), 2017. [Consultado el 05 de marzo de 2018]. URL disponible en: http://bvs.minsa.gob.pe/local/MINSA/4146.pdf.

8. De La Torre Rendón, F. Lesión premaligna escamosa del cuello uterino, un enfoque actualizado [Revista en línea]. Patología - Revista Latinoamericana. 2008;46(4):332-42. [Consultado el 05 de marzo de 2018]. URL disponible en:

http://www.medigraphic.com/pdfs/patrevlat/rlp-20 08/rlp084g.pdf

9. Vega A, María. Factores de riesgo para cáncer cervicouterino en mujeres de Matamoros, Tamaulipas. Universidad Autónoma de Nuevo León [Sede Web].

México 2004. [Consultado el 05 de marzo de 2018]. Disponible en: http://eprints.uanl.mx/5407/1/1020149340.PDF

10. Análisis de la situación del cáncer en el Perú [En línea]. Dirección General de Epidemiología. Lima (Perú), 2013. [Consultado 29 de noviembre de 2016] URL disponible en:

http://www.dge.gob.pe/portal/docs/asis_cancer.pdf.

11. Henríquez Handrea M., Ortega Bonilla R. Factores de riesgos en mujeres diagnosticadas con en el hospital José Nieborowski, Boaco. enero 2013-diciembre 2014 lesiones pre malignas de cuello uterino a través de PAP, atendidas [Tesis doctoral en línea]. Boaco, Nicaragua: Facultad de Ciencias Médicas de la Universidad Nacional Autónoma de Nicaragua. 2015. [Consultado el 05 de marzo de 2018]. URL disponible en: http://repositorio.unan.edu.ni/1485/1/41998.pdf

12. Aguilar Palomino G.R. Factores de riesgo asociados a cáncer de cérvix en pacientes atendidas en el hospital Vitarte durante el año 2015 [Tesis de grado en línea]. Lima (Perú): Facultad de Medicina Humana de la Universidad Ricardo Palma. 2017. [Consultado el 05 de marzo de 2018]. URL disponible en: 
http://cybertesis.urp.edu.pe/bitstream/urp/932/1/Ag uilar\%20Palomino\%20Gabriela\%20Raquel_2017.pdf

13. Bonilla Tello K.A., Montalvo De La Torre Y.I. Factores de riesgo relacionados a lesiones pre malignas de cáncer del cuello uterino. hospital Victor Ramos Guardia Huaraz 2008 - 2012 [Tesis de grado en línea]. Huaraz (Perú): Facultad de Ciencias Médicas de la Universidad Nacional "Santiago Antúnez De Mayolo". 2015. [Consultado el 05 de marzo de 2018].

14. Smith J.S., Green J., Berrington A., Appleby P., Peto J., Plummer M., Franceschi S., Beral V., Cervical cancer and use of hormonal contraceptives: a systematic review [Revista en línea]. Lancet. Estados Unidos, abril 2003. 361(9364):1159-67. [Consultado 29 de noviembre de 2016] URL disponible en: https://www.ncbi.nlm.nih.gov/pubmed/12686037.

15. Rodríguez G., Barrios E., Vasallo J. Características epidemiológicas de una población que accedió al programa de prevención del cáncer de cuello uterino en Uruguay. Rev Med Uruguay 2005; 21: 200-206

16. García Minaya, O. Principales factores de riesgo del cáncer de cuello uterino main risk factors of uterine cervical cancer". In Crescendo Ciencias de la salud, [S.I.], v. 4, n. 2, p. 609-616, ene. 2018. [Consultado el 28 de febrero de 2018]. URL disponible en: $<$ http://revistas.uladech.edu.pe/index.php/increscend o-

17. Ortiz Serrano R., Uribe Pérez C.J., Díaz Martínez L.A., Dangond Romero R.Y., Factores de riesgo para cáncer de cuello uterino [En línea]. Scielo. 2004. [Consultado 30 jun 2018]; 55(2) Disponible en: http://www.scielo.org.co/scielo.php?script=sci_arttext \&pid=S0034-74342004000200007

18. Fernández Velásquez C.C., Izaguirre Villanueva E. Factores de riesgo socioeconómicos y gineco obstétricos que predisponen a cáncer de cuello uterino [Tesis de grado en línea]. Nuevo Chimbote (Perú). Facultad de Ciencias de la Universidad Nacional de Santa. 2014. [Consultado el 05 de marzo de 2018]. URL disponible en:

http://repositorio.uns.edu.pe/bitstream/handle/UNS/ 1917/26307.pdf?sequence =1\&isAllowed =y

19. Gómez Sifuentes J.L. Factores de riesgo para cáncer de cuello uterino. hospital Regional docente de Trujillo (HRDT) 2007-2012 [Tesis de grado en línea]. Trujillo (Perú): Facultad de Medicina de la Universidad Nacional de Trujillo. 2013. [Consultado el 05 de marzo de 2018]. URL disponible en:

http://dspace.unitru.edu.pe/bitstream/handle/UNITR U/627/GomesSifuentes_J.pdf?sequence=1\&isAllowed $=\mathrm{y}$

20. Thulaseedharan J, Malila N, Hakama M, Esmy $P$, Cheriyan M, Swaminathan R, et al. Socio demographic and reproductive risk factors for cervical cancer - a large prospective cohort study from rural India. Asian Pac J Cancer Prev. 2012;13(6):2991-5.

21. Cabrera Guerra I., Ortiz-Sánchez Y., Suárez Gómez Y., Socarrás Rodríguez R., Vázquez-Rosales N. Factores de riesgo asociados al cáncer cérvico-uterino en el área de salud de Santa Rita [En línea]. MULTIMED Revista Médica Granma. Granma (Cuba), 2017. [Consultado el 05 de marzo de 2018]. URL disponible en:

http://www.revmultimed.sld.cu/index.php/mtm/artic le/view/392

22. Salazar Rodríguez S.K. Relación entre el nivel de conocimiento sobre cáncer cervical y la exposición a sus factores de riesgo en usuarias atendidas en los consultorios externos del Instituto Nacional Materno perinatal, febrero - marzo del 2016 [Tesis de grado en línea]. Lima (Perú): Facultad de Medicina de la Universidad Mayor de San Marcos. 2016. [Consultado el 05 de marzo de 2018]. URL disponible e $\mathrm{n}$

http://cybertesis.unmsm.edu.pe/bitstream/handle/c ybertesis/4760/Salazar_rs.pdf;jsessionid=40B44D1D 801D9A42858C00466281300A? sequence $=1$

23. Domínguez E. Factores de riesgo para cáncer cérvico uterino en pacientes atendidas en el Centro Estatal de cancerología dr. miguel dorantes mesa. méxico. 2012.

24. Sotomayor Y. Conocimiento y actitudes respecto al cáncer cervicouterino en las mujeres atendidas en el Hospital Sabogal. Lima.2008.

25. Raychaudhuri S, Mandal S. Socio-demographic and behavioural risk factors for cervical cancer and knowledge, attitude and practice in rural and urban areas of north Bengal, India. Asian Pac J Cancer Prev. 2012;13(4):1093-6.

26. Appleby $P_{\text {., }}$ Beral V., Berrington A., Colin D., Franceschi S., Goodhill A., Green J., Peto J., Plummer M. cervical cancer and hormonal contraceptives: collaboratie collaborative reanalysis of individual data for 16,573 women with cervical cancer and 35,509 women without cervical cancer from 24 epidemiological studies [Revista en línea]. Lancet. Estados Unidos, nov 2007. 370(9599):1609-21. [Consultado 29 de noviembre de 2016] URL disponible en: https://www.ncbi.nlm.nih.gov/pubmed/17993361.

27. Dunán Cruz L.K., Cala Calviño L., Infante Tabío N.I., Hernández Lin $T$. Factores de riesgo ginecoobstétricos para el cáncer cervicouterino en la atención primaria de salud [en línea]. Medisan. Cuba, mayo 2011. 15(5): 573-579. [Consultado 29 de noviembre de 2016] URL disponible en: http://scielo.sld.cu/scielo.php?script=sci_arttext\&pi $d=$ S1029-30192011000500002.

28. Conde Félix A.M. Factores de riesgo, asociados a lesiones en el cuello uterino, en mujeres en edad fértil Hospital María Auxiliadora [Tesis de grado en línea]. Lima (Perú): Facultad de Obstetricia y Enfermería de la Universidad Mayor de San Marcos. 2014. [Consultado el 05 de marzo de 2018]. URL disponible en:

http://www.repositorioacademico.usmp.edu.pe/bitst 
ream/usmp/1509/1/Conde_fa.pdf

29. Coaquira Galindo M.P., Curitomay Yanqui, R. Factores de riesgo de las neoplasias cervicales intraepiteliales estudiadas en la dirección regional de salud de Ayacucho y hospital "Victor Ramos Guardia" de Huaráz en el año 2001 [Tesis de grado]. Ica (Perú): Facultad de Medicina Humana de la Universidad Nacional "San Luis Gonzaga" de Ica. 2002

30. Cabrera Arenas, J.C. Distribución de los factores de riesgo en el cáncer de cuello uterino en pacientes del Hospital Nacional Sergio e. Bernales, 2006-2011 [Tesis de grado en línea]. Lima (Perú): Facultad de Medicina Humana de la Universidad Mayor de San Marcos. 2012. [Consultado el 05 de marzo de 2018]. URL disponible en:

http://cybertesis.unmsm.edu.pe/bitstream/handle/cy bertesis/452/Cabrera_aj.pdf? sequence $=1$

31. Factores de riesgo de cáncer cervicouterino: indicios hasta la fecha [Sede en línea]. Nueva York: Alliance for Cervical Cancer Prevention; 2004 [Consultado 28 de febrero de 2018]. URL disponible en: http://screening.iarc.fr/doc/RH_risk_factors_fs_sp.pd

32. Píldoras anticonceptivas y el riesgo de cáncer [En línea] Estados Unidos: Instituto Nacional del Cáncer; 2007 [Actualizado 2014, Consultado 30 ene 2018]. URL disponible en: https://www.cancer.gov/espanol/cancer/causas-pre vencion/riesgo/hormonas/hoja-informativa-pildoras -anticonceptivas\#r15

33. Salinas Tomalá J.A., Villarroel Peña G.E. Factores de riesgo y prevención del cáncer cérvico uterino en las usuarias de la "clínica granados". salinas. 2011-2012 [Tesis de grado en línea]. La Libertad (Ecuador): Facultad de Ciencias Sociales y de la Salud de la Universidad Estatal Península de Santa Elena. 2012. [Consultado el 05 de marzo de 2018]. URL disponible en:

http://repositorio.upse.edu.ec/bitstream/46000/618 11/SALINAS\%20TOMALA\%20JANETH.pdf

34. Lobo Abascal P. Anticoncepción hormonal y riesgos de cáncer de mama, ovario, endometrio, cérvix y otros [En línea]. Hospital Universitario Infanta Sofía. Madrid (España), 2011. [Consultado 29 de noviembre de 2016] URL disponible en: http://sec.es/descargas/PS_Anticoncepcion_hormon al_y_riesgo_de_cancer.pdf.

35. Bosch F.X., Manos M.M., Muñoz M., Sherman M., Jansen A.M., Peto J., et al. Prevalence of human papillomavirus in cervical cancer: a worldwide perspective. International Biological Study on Cervical Cancer (I BSCC) Study Group. J Natl Cancer Inst 1995;87:796-802.

\section{Las ediciones anteriores de revista médica PANACEA están disponibles en:}

\section{www.revpanacea.unica.edu.pe}

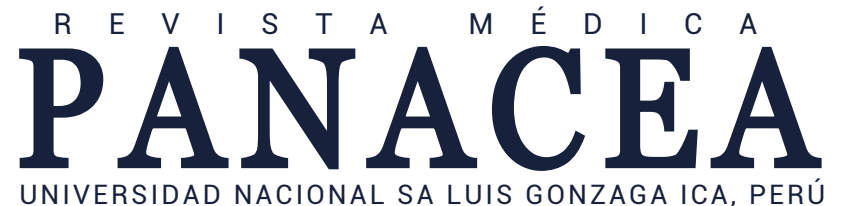

